\title{
Cesária em paciente portadora de miocardiopatia hipertrófica
}

- Andréia Miranda de Carvalho

- Roseny Reis Rodrigues

- Filomena Regina B. G. Galas

\author{
Instituto do Coração do Hospital das Clínicas, Faculdade de Medicina \\ da Universidade de São Paulo, São Paulo
}

INTRロDUÇÃa

Uma gestação promove alterações fisiológicas acentuadas no organismo materno e, quando temos essas duas situaçôes combinadas, miocardiopatia hipertrófica e gestação, vários agravantes devem ser levados em consideração antes que se realize qualquer técnica anestésica para a cesariana.

RELATO DO CASD

Gestante, 23 anos (gesta 4 para 3 aborto 1), $60 \mathrm{~kg}$, com idade gestacional de 37,6 semanas, chega ao pronto-socorro com a queixa principal de dispnéia aos pequenos esforços e palpitação. Ao exame físico, apresenta-se em estado geral regular, descorada $(+/ 4+)$, hipohidratada $(+/ 4+)$, eupnéica, sem sinais de estase jugular ou de edema de membros inferiores. Ausculta cardíaca com bulhas normofonéticas, $2 \mathrm{~T}$ com sopro sistólico 4+/6+ panfocal. Ausculta pulmonar normal. Ecocardiograma: $\mathrm{FE}=78 \%$; espessura de septo = $33 \mathrm{~mm}$; gradiente máximo de $\mathrm{VE}=129$; insuficiência mitral de grau leve e hipertrofia septal assimétrica com importante obstrução de saída de VE. Três dias após a internação, a paciente apresentou piora do estado geral, com dispnéia importante e desmaio. Optou-se por induzir o parto. Juntamente à indução, foi realizada analgesia peridural. Iniciou-se a administração de uma solução analgésica em bomba de infusão contínua. A solução peridural conteve bupivacaína a $0,5 \%$ com adrenalina (4 ml), sufentanil $10 \mathrm{mcg}$ e água destilada $(34 \mathrm{ml})$.Cinco horas após a instalação do bloqueio peridural, a paciente foi levada para a sala de operação, a qual chegou acordada, consciente, com bloqueio motor completo e bloqueio sensitivo em T10. Devido ao nível de anestesia insuficiente, optou-se pela anestesia geral balanceada em seqüência rápida com manobra de Sellick. Após monitorização, foram utilizados na indução: etomidato $(18 \mathrm{mg})$, fentanil $(50 \mathrm{mcg})$ e succinilcolina $(60 \mathrm{mcg})$. Depois de $15 \mathrm{~min}$ e retirada do $\mathrm{RN}$ único, vivo, foram administrados atracúrio (30 mg) e propofol $(20 \mathrm{mg})$. A paciente manteve-se estável durante o procedimento, mantendo $\mathrm{PA}=100 / 50 \mathrm{mmHg}$, $\mathrm{SpO}_{2}=100 \%$ e $\mathrm{PetCO}_{2}=35$. Ao final da cesariana, a paciente foi extubada sem intercorrências e levada para a UTI acordada, hemoestável, sem uso de drogas vasoativas. Cinco dias depois da cirurgia, a paciente recebeu alta hospitalar assintomática, em uso de propranolol, verapamil e sulfato ferroso.

DIScUSรÃa

Uma das mais importantes preocupaçôes na miocardiopatia hipertrófica em gestante é se há aumento do risco de morte. A anestesia peridural não tem sido geralmente aconselhada. Algumas complicações foram relatadas. O mecanismo da hipotensão arterial e/ou tontura não ficaram claros. Podem ser atribuídos à exacerbação da resposta normal à anestesia peridural ou à piora do gradiente do fluxo ventricular esquerdo. Por isso, até que mais informações se tornem acessíveis, a anestesia peridural deve continuar sendo aplicada cautelosamente em pacientes que possuem obstrução de fluxo de saída.

REFERÊNCIAS

1. Ho,Kwok M MRCP, et al. Combined spinal and epidural anesthesia in a parturient with idiopathic hypertrophic subaortic stenosis. Anesth. 1997;187:168-9.

2. Thaman R, et al. Pregnancy related complications in women with hypertrophic cardiomyopathy. Heart. 2003;89:752-6.

\footnotetext{
Endereço para correspondência:

Andréia Miranda de Carvalho

Rua Capote Valente, 493 - apto. 11 - Pinheiros

São Paulo (SP) - CEP 05409-001

Tel. (+55 11) 3081-5571

E-mail: andreiagat@yahoo.com.br
} 